LAWRENCE LIVERMORE N A T IO N A L LABORATORY

\title{
21st Century Steam for Asteroid Mitigation
}

D. S. Dearborn

March 16, 2004

2004 Planetary Defense Conference Protecting Earth from Asteroids

Orange County, CA, United States

February 22, 2004 through February 26, 2004 
This document was prepared as an account of work sponsored by an agency of the United States Government. Neither the United States Government nor the University of California nor any of their employees, makes any warranty, express or implied, or assumes any legal liability or responsibility for the accuracy, completeness, or usefulness of any information, apparatus, product, or process disclosed, or represents that its use would not infringe privately owned rights. Reference herein to any specific commercial product, process, or service by trade name, trademark, manufacturer, or otherwise, does not necessarily constitute or imply its endorsement, recommendation, or favoring by the United States Government or the University of California. The views and opinions of authors expressed herein do not necessarily state or reflect those of the United States Government or the University of California, and shall not be used for advertising or product endorsement purposes. 


\title{
$21^{\text {st }}$ Century Steam for Asteroid Mitigation
}

\author{
by \\ D S P Dearborn \\ Lawrence Livermore National Laboratory
}

"Who knows whether, when a comet shall approach this globe to destroy it, as it often has been and will be destroyed, men will not tear rocks from their foundations by means of steam, and hurl mountains..." Medwin's Conversations of Lord Byron

\begin{abstract}
:
The systematic requirements to divert an object on an earth-impacting course are developed relating the minimum velocity perturbation (both magnitude and direction) to the time available before impact. This, coupled with the accuracy to which orbits can be determined, restricts the time available for any mitigation technology to operate. Because nuclear energy densities are nearly a million times higher than those possible with chemical bonds, it is the most mass efficient means for storing delivering energy with today's technology. The question is how to most effectively apply that energy. This paper will examine the simple case of shattering the body, as well as a more controlled approach in which one or more small velocity increments divert a body. The optimal approach depends on the detailed circumstances, but in either case, already developed technology permits a successful diversion with a few years to decades of notice. The success of nuclear options on relatively short timescales permits consideration of other technologies that while not so well developed might be sufficiently improved to divert small (100 meter) bodies.
\end{abstract}




\section{Introduction}

Though rare, terrestrial impacts by sizable objects $(>1000 \mathrm{~m})$ are potentially catastrophic, and as the earth's population density increases, the threat posed by even smaller bodies becomes significant. In addition to the direct threat on urban population centers, such impacts can have broad reaching environmental consequences, disrupting the complex social and economic interactions that support modern population densities.

Fortunately, we have achieved a level of sophistication that enables us to recognize and respond to such a long-term threat. Discovery and monitoring efforts to find and track asteroids promise to provide decades to centuries of warning of the most threatening bodies. However, the precision to which tracking and orbit prediction can operate constrains real mitigation strategies.

Elaborating on this point, figure 1shows the orbital uncertainty in the position along its track (measured in earth radii per year) for about 40 asteroids. As expected, the longer the object has been observed, the better the orbital period is known, and so its position along its track. By 10 orbital periods, the uncertainty is a few thousandths of an earth radius per year, suggesting that NEO's, observations of 10 to 20 years are sufficient to predict an impact many centuries out. Objects that pass near enough the earth to be observed by radar require even less time to be confirmed as a threat.

Figure 1) Using published orbits for objects with different observing histories, this figure shows the decrease in orbital uncertainty with time.

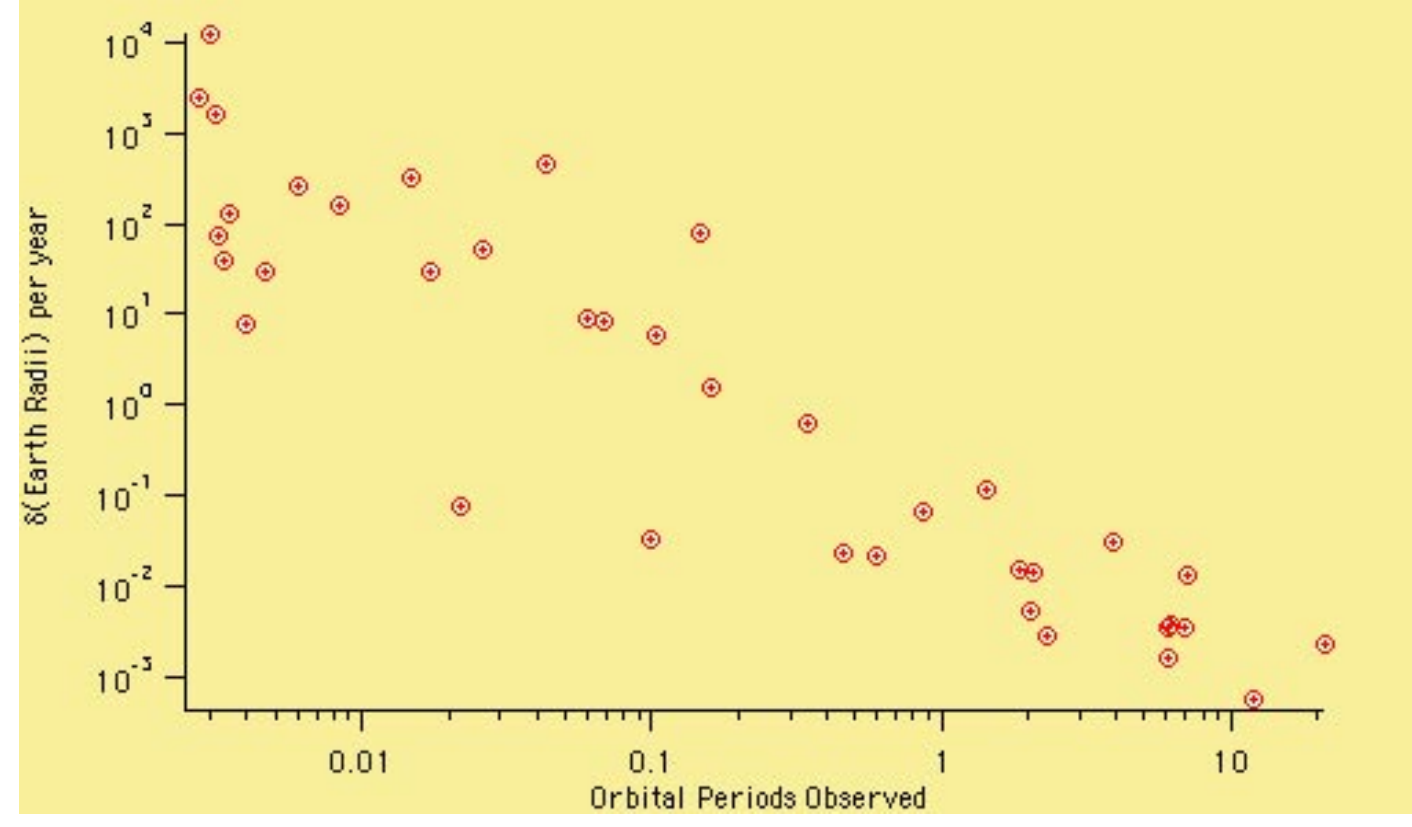

Unfortunately, over centuries of time, both gravitational (near passages of planets) and non-gravitational ( solar wind and flux) interactions limit the precision with which we can predict the fate of even a well observed body. An excellent example of this problem is presented by Giorgini, et al (2002) for the body DA 1950.

DA 1950 is a slightly asymmetrical spheroid with a mean diameter of $1.1 \mathrm{~km}$, rotating 
once every 2.1 hours. Its eccentricity is about 0.507 , the semimajor axis $1.69966 \mathrm{AU}$ and the orbit is inclined12.184 ${ }^{\circ}$. It was first discovered on 23 Feb 1950, and rediscovered at Aricebo (radar) in March of 2001, when the body passed within $0.05 \mathrm{AU}$ of earth. With a 50-year base of observation and the precision of radar detection, this orbit is about as well determined as is possible, and it is interesting in that there is a non-zero collision probability on Saturday 16 March $2880 \mathrm{AD}$, in the range $0.33 \%$. The authors of that paper present a detailed error analysis showing how a number of effects impact the ability to predict the fate of this object over the coming 8 centuries.

\begin{tabular}{lrr} 
Along-track effect & Distance $\left(\mathrm{R}_{\text {earth }}\right)$ & Time \\
- \hdashline-1.3 & $-10 \mathrm{~min}$ \\
Galactic tide & -1.6 & $-12 \mathrm{~min}$ \\
Numerical integration error & +2.1 & $+16 \mathrm{~min}$ \\
Solar mass loss & +6.5 to +2.8 & +49 to $+21 \mathrm{~min}$ \\
Solar oblateness (J2) & -235 & -1.2 days \\
61 additional asteroids & +215 to -240 & +1.1 to -1.3 days \\
Planetary mass uncertainty & $-1,750$ & -9.1 days \\
Solar radiation pressure & $-1,725$ to $-2,760$ & -9.0 to -14.0 days \\
Combined above & $+1,870$ to $-11,000$ & +9.6 to -57.0 days \\
Yarkovsky effect only &
\end{tabular}

The most serious effects noted in that paper are solar radiation and the Yarkovsky effect (re-radiation of the absorbed solar flux in a preferred direction). Light absorbed by the body surface provides a radial momentum transfer, and the bodies motion through the photon field results in aberration, and a Doppler component that slows the body. Because the force depends on the area of the object, while its mass depends on the volume, the acceleration scales roughly as the inverse radius of the body, making these processes even more serious for small (100 meter) bodies.

The magnitude of these radiation interactions depends on the surface composition and structure as well as the rotation rate and direction. As most asteroids are irregularly shaped, with off axis moments, predicting long-term orientation is a complicate the problem. The albedo (which may be variable over the surface), as well as thermal conduction/storage properties are very difficult to constrain from remote sensing. It is unlikely that a sufficiently precise orbit will be available for DA 1950 for centuries to come.

By a century or so before the possible impact date, these non-gravitational terms will no longer be significant, and the error associated with close planetary encounters reduced so that a precise determination of the 16 March encounter can be made. Until the certainty of an impact is very high, it will be difficult to consider any mitigation action. First, when the error ellipsoid is large compared to the earth, the mitigation technology must either change the orbit by a large amount such that the entire ellipsoid misses, or risk converting a non-impacting orbit into an impacting one. A second, less technical but real challenge to any action prior to obtaining a high degree of certainty of an impact is to convince society to expend the very significant amount of resources to divert a possible non-threat. 
The problem of predicting a certain collision over long periods, makes it is necessary to examine mitigation strategies that can operate in decades to something of order a century. This comes down to determining the magnitude and direction of the velocity perturbations necessary to divert an earth-impacting asteroid from its natural finale. It is generally understood that the work to be done decreases when a perturbation is applied early. For some orbits, very small velocity changes $\left(10^{-4} \mathrm{~m} / \mathrm{s}\right)$ may be sufficient to divert a collision, if it is applied many decades before the impact. However once an impulse is imparted to the body, the velocity/orbit must be re-determined in a time that is short compared to the remaining time to impact, to assure that the diversion was successful. For tiny velocity perturbations, the necessary precision necessary is high, and time to make the determination is limited. In summery, the limits of the observation technology to quickly determine a new orbit, sets a minimum robustness level for any mitigation strategy even after the impact certainty is high.

\section{Mitigation Strategies:}

Many clever proposals have been made for methods to divert an asteroid. Some, like a recent suggestion to change the albedo of the body with the early investment of 250 tons of white paint, fail to operate in a short enough period to be other than a gamble as to whether they will cause an impact or stop one. however, some general requirements can be set for the process of asteroid diversion.

Newton's laws of motion place certain immutable requirements on changing the motion of a body. As an example (Table 1), an object with a 0.7 year period and an eccentricity of 0.65 requires a speed change of $0.01 \mathrm{~m} / \mathrm{s}$ if applied 7 to 35 years prior to impact (depending on orbital phase as will be addressed later). Momentum conservation sets a relation between the ejecta mass, ejecta velocity and the asteroid response that applies to any method.

$$
\frac{\delta M_{\text {ejecta }}}{M_{\text {asteroid }}}=\frac{\delta V}{V_{\text {ejecta }}}
$$

For an asteroid mass of $10^{12} \mathrm{~kg}$, typical of a $1 \mathrm{~km}$ body, an ejecta velocity, $\mathrm{V}_{\text {ejecta }}$, of 1 $\mathrm{km} / \mathrm{s}$ (typical of chemical propellants) requires heating $10^{4}$ tons of reaction mass carrying $1 \mathrm{Kt}$ of kinetic energy.

Table 1: Newton Says let there be momentum conservation

\begin{tabular}{ccclcc} 
Asteroid & Asteroid & Ejecta & \multicolumn{2}{c}{ Reaction } & \multicolumn{2}{c}{ Time to Impact } \\
$\mathrm{\delta V}$ & Energy & Energy & Mass & perihelion & aphelion \\
$\mathrm{m} / \mathrm{s}$ & tons & $\mathrm{Kt}$ & Tons & days & days \\
0.01 & 0.01 & 1. & $10^{4}$ & 2507 & 12900 \\
0.1 & 1.0 & 10. & $10^{5}$ & 250 & 1293 \\
1.0 & 100.0 & 100. & $10^{6}$ & 25 & 129 \\
10.0 & $10,000.0$ & 1000. & $10^{7}$ & 3 & 12
\end{tabular}

The high exhaust velocities of an ion drive may reduce the reaction mass requirement by a factor of 100 , but it increases the minimum energy budget by the square of that factor. Since the fuel is not, itself, a high energy density material, electricity to 
accelerate the ions must be provided by other means. Thruster approaches must also use fuel to match the orbit of the body, and subsequently deal with any rotation and attachment issues.

If an asteroid were sufficiently structurally sound, simply impacting this mass on the surface (in small pieces) will transfer momentum to the body to perturb the velocity. In this example, a closing velocity of $10 \mathrm{~km} / \mathrm{s}$, requires impacting 1000 tons to change the velocity by $0.01 \mathrm{~m} / \mathrm{s}$. At these speeds, the kinetic energy per kilogram is greater than that in high explosive, and the impacts will act like explosions. In a rigid body, the backscatter of material from the crater-forming region might increase the momentum substantially, but even a factor of 10 requires a total of 100 tons of impacting material. As large asteroids are not thought to be solid rocks with mechanical rigidity, this momentum multiplication cannot be depended on, and is a major uncertainty.

Ablative proposals, (lasers, particle beams, ...) avoid carrying the reaction mass with them, and utilize the asteroid's own material. They must still meet the energy requirements for the ejecta, and, in addition, there is an energy cost to vaporizing the surface material. Continuing the above example, $>5 \mathrm{Kt}$ of additional energy is needed to heat and vaporize the surface material (assuming it has the properties of olivine). If this energy were delivered by photons or neutral particles, it must be delivered as intense pulses to avoid self-shielding (and defocusing) of the beam by the material blow-off. It is a substantial technical challenge to develop a laser capable of radiating $>5 \mathrm{Kt}$ in a pulsed mode with the high spectral purity required for precise focus. The focus requirement arises because the energy concentration necessary to ablate material is too high for the optical elements of a laser, so the beam must be focused to the target distance. The National Ignition Facility (NIF) will be able to produce laser pulses with an infrared energy of 4 megajoules, requiring more than 5 million pulses to deliver the necessary energy. At the current designed shot rate this would require over 6000 years to avoid damaging the laser from thermal loading. To deliver this energy in 2 months near perihelion passage will require improving the firing rate from a few times per day to once per second (A rate increase of 30,000). Other laser schemes might be preferred for asteroid deflection, but achieving all necessary conditions (efficiency, purity, total throughput) will require significant development.

Nuclear reactions are nearly a million-times more energetic than chemical, and nuclear devices of less than a ton carry far more energy than is required to accelerate a $10^{12} \mathrm{~kg}$ body by $10 \mathrm{~m} / \mathrm{s}$. The question is how to optimally utilize such an energy source. We will show that with just a few years warning, a sub-kilometer size object (like the Athos case of the planetary defense conference) simply attacked in the best Hollywood fashion is dispersed over a volume over a hundred of thousands times that of the earth. The fraction debris that remains on an impacting trajectory carries negligible energy, but would have to be examined to provide assurance that none of the remaining pieces pose a threat. As the size approaches a kilometer and when time permits, a more thoughtful application of force is warranted. We will discuss how larger objects can be impulsively accelerated with small velocity increments sufficient to avoid an impact, while the bulk of the material remains gravitationally bound as a single object (even if there is no material strength).These applications of nuclear energy ate the main subject of this paper. 
In the following sections we provide a short summery of the physics of asteroid motion in the solar system to see how orbits evolve from non-hazardous paths to ones that support an impact. Deterring an impact can involves modifying the orbit so that it is no longer hazardous, or delaying the impact threat until the orbit naturally evolves to nonhazardous. With this information, it is possible to systematically examine the result of different velocity perturbations on a range of orbital elements, and to develop a relationship between the magnitude of the velocity perturbation and the time at which it is applied. Finally, we examine technologies for achieving those perturbations, in particular well developed nuclear technologies.

\section{Orbit crossing versus intersection:}

When viewed on the ecliptic plane (figure 2), the orbit of an asteroid may appear to intersect the orbit of the earth, but orbital inclination and orientation generally assures that neither of crossing is a true intersection. However, the concentration of planets near the ecliptic forces a slow precession that changes the obit from one that does not actually intersect the earth's to one that does. In addition, NEO orbits are influenced by close encounters with planets (figure 3 ), resulting in sharp discrete changes in orbital elements. Both of these processes operate to evolve an orbit.

Fig 2a: View from above ecliptic showing 27 earth orbits and nearly 40 asteroid orbits.

Fig 2b: View from plane of ecliptic
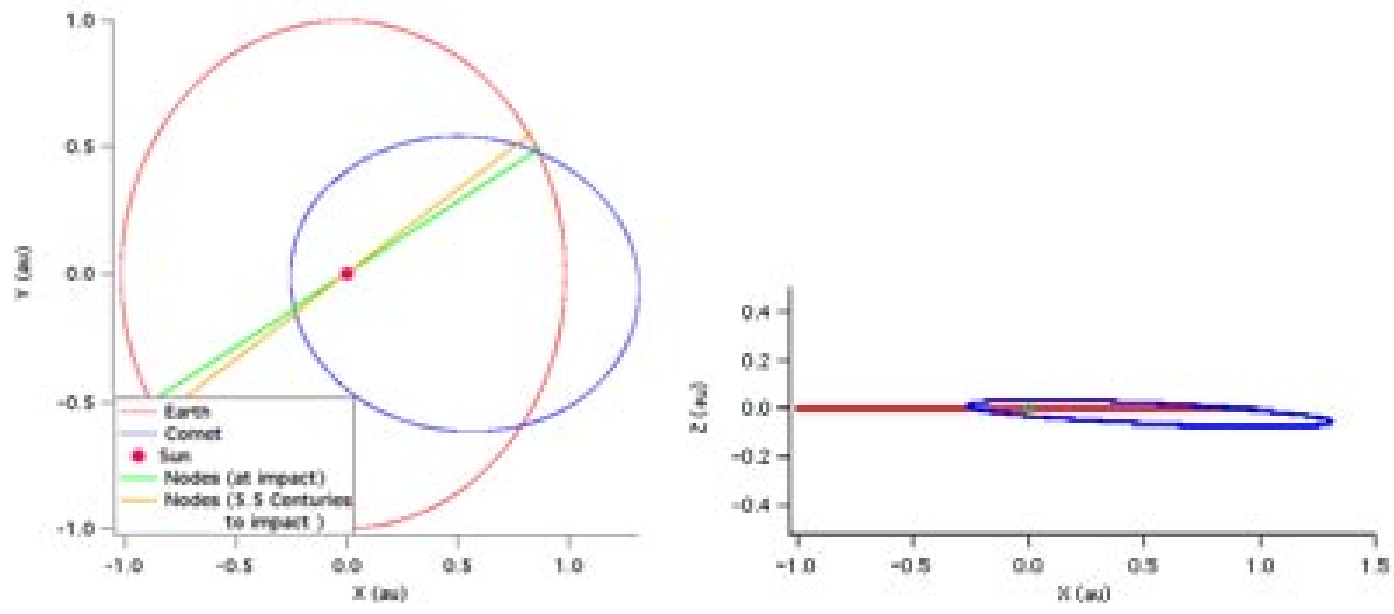

Fig 3: Over the course of 40 orbits, sharp changes in the inclination (aw well as semimajor axis and eccentricity) are seen to coincide with the closer encounters between the earth and asteroid.

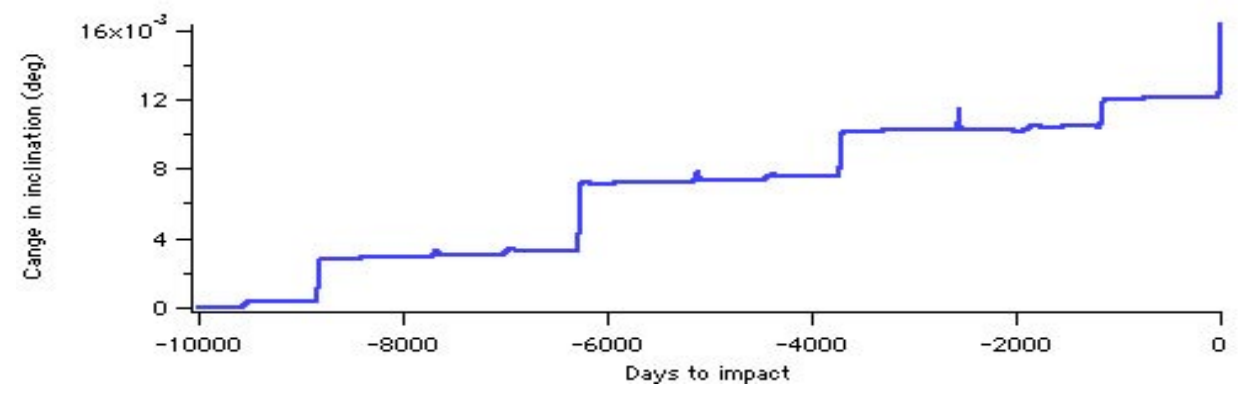


Once the orbits are intersecting, a collision is still not assured. Only if the phase of the asteroid and earth are such to bring them to the intersection point at the same time will there be a collision. If a collision is deferred, precession, and close passages will cause the orbits to move apart. In the mean time, there is a period of hazard when collisions can occur. Mitigation strategies can operate to either modify phase, in which case attention must be paid to make sure that the impact does not occur on a different orbit, or the orbit can be precessed beyond the intersection.

\section{Systematic survey of mitigation by velocity perturbations:}

The reference frame defined by the motion of an asteroid is the natural one in which to consider applying velocity perturbations, and in this section we use the $\mathrm{T}, \mathrm{N}$, and $\mathrm{W}$ frame (figure 4) as defined below:

$\mathrm{T}+/$ - speed: aligned with direction of motion [v].

$\mathrm{N}+$ - yaw: orthogonal to direction of motion but in the plane of orbit [ $v X(v X r)$ ].

W +/-pitch: orthogonal to the direction of motion and the orbital plane [v $\mathrm{Xr}$ ].

Fig 4: Angular momentum is an important defining element of an orbit.



Considering the orbit in terms of its angular momentum provides a simple understanding of how various velocity perturbations will modify the orbit. Velocity perturbations applied in the $\mathrm{T}$ direction (either + or -) change the magnitude of the angular momentum vector but not orientation. As a result, the period increases or decreases, and an object on a collision path loses phase. The more orbits that pass before the expected collision, the more de-phasing that occurs, i.e. the miss distance increases with time (figure 5). The magnitude of the miss is dependant on the phase at which the perturbation is applied, as will be discussed below. 
Fig 5: The miss distance is shown for velocity perturbations applied along the $T$ direction (direction of motion), between 10 and 10,000 days prior to impact. The largest reaction occurs for impulses applied at perihelion and the smallest at aphelion.

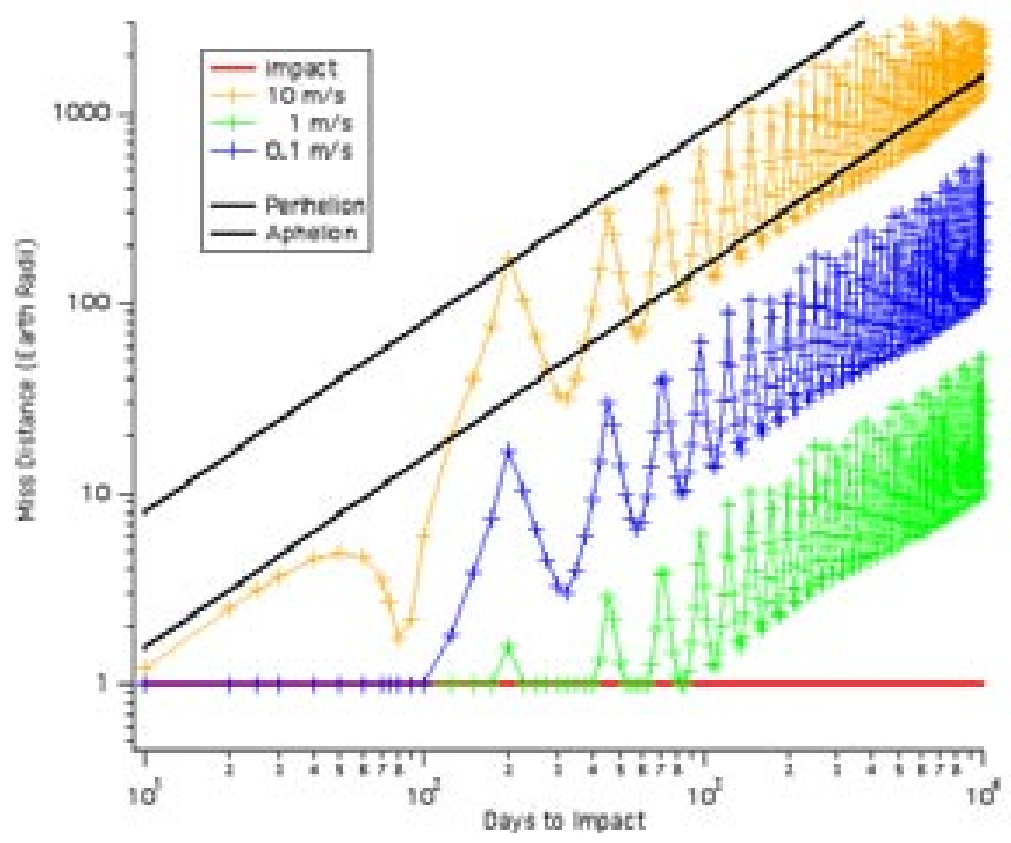

The force necessary to produce a velocity perturbation in the $\mathrm{W}$ direction provides a torque that will precess the orbit. This changes the longitude of the ascending node, and the arguement of perihelion passage. It can also modify the inclination slightly. If the orbital precession is sufficient to change the orbit from a intersecting to nonintersecting, a collision is avoided.

Fig. 6: Miss distances for a $10 \mathrm{~m} / \mathrm{s}$ velocity perturbations applied in the $\mathrm{W}$ direction, orthogonal to both the orbital plane and the direction of motion.

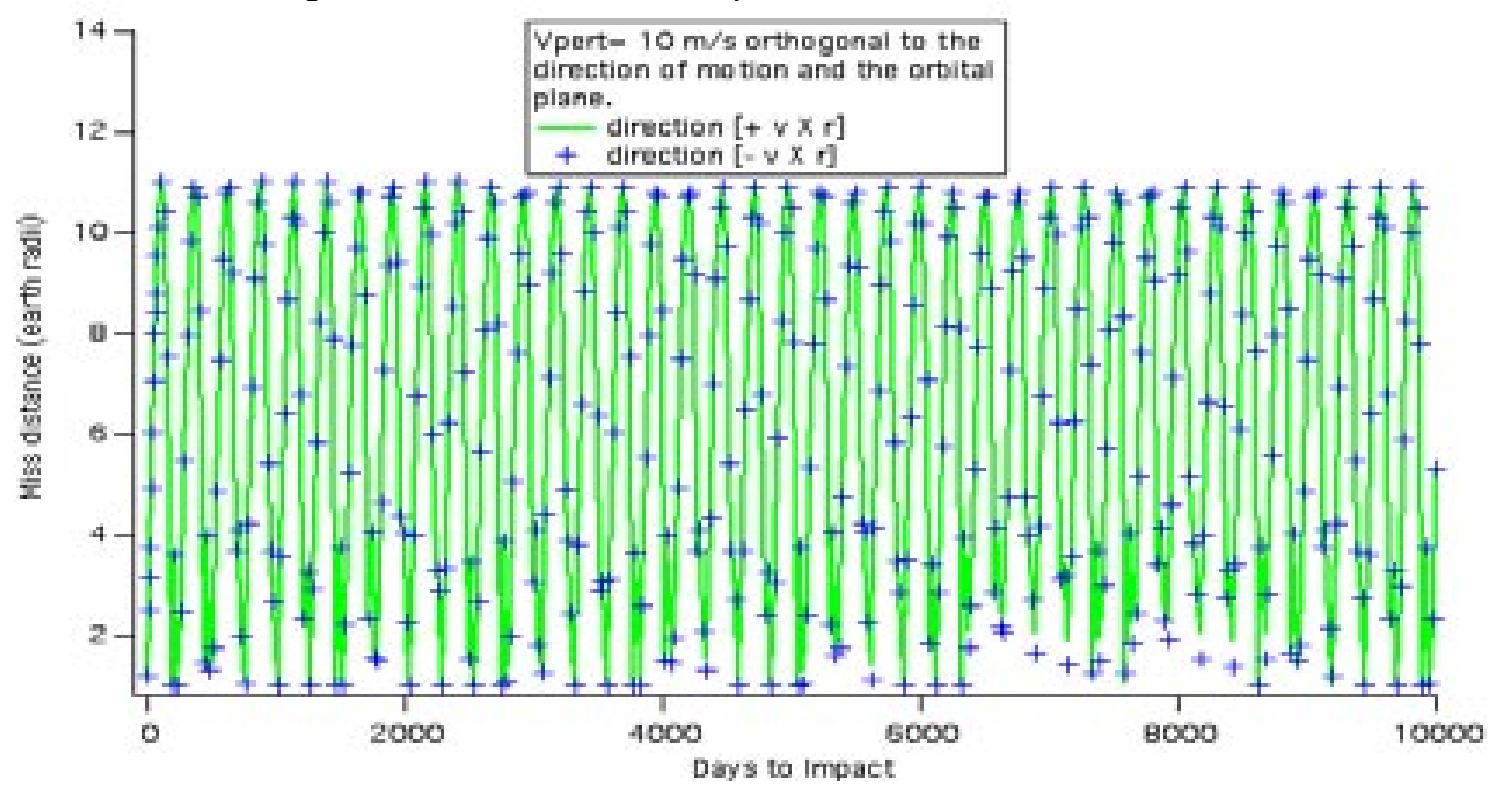


In the absence of close approaches to planets, there is no growth in the miss distance with early application (figure 6), but there is a phase sensitivity here too. Because the miss distance does not automatically grow with time, the magnitude of the velocity perturbation necessary to deflect an impact is relatively high. Perturbations in this direction should be considered primarily when a body is discovered at a relatively late time, and this direction is found to require a lesser impulse for deflection than the other directions.

Finally, velocity perturbations in the $\mathrm{N}$ direction changes eccentricity and rotates orbit (changes the arguement of perihelion passage). As with the $\mathrm{W}$ direction, perturbations here can convert an orbit from intersection to non-intersecting, but the miss magnitude does not intrinsically grow with time. In the circumstance that the colliding orbit involves a series of well-defined close approach to the earth or another planet, those passages can be used to magnify or de-magnify the resulting miss (figure 7). Again, the magnitude of the required perturbation is relatively large and can be recommended only in special circumstances.

Fig 7: Miss distances for a $10 \mathrm{~m} / \mathrm{s}$ velocity perturbations applied in the $\mathrm{N}$ direction, lying in the orbital plane and orthogonal to the direction of motion

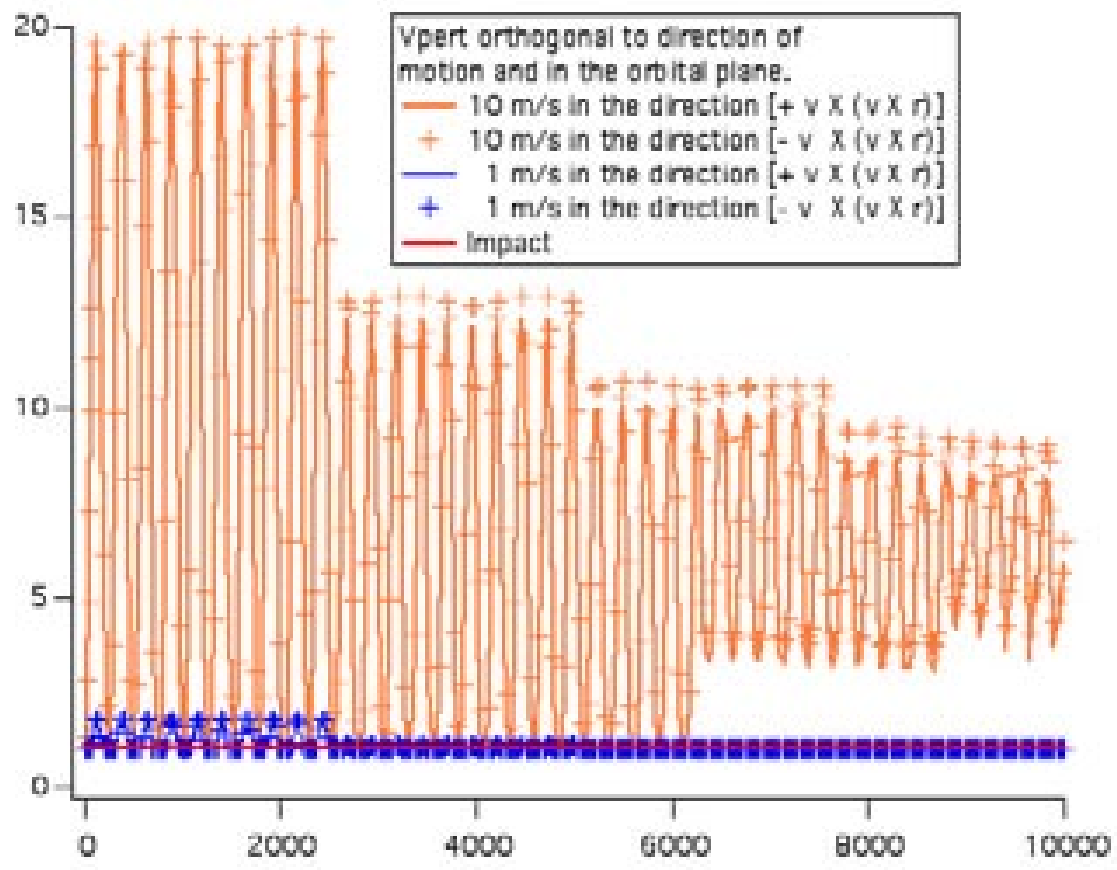

\section{Minimum conditions for diverting an asteroid via impulse:}

As discussed, speed changes along the direction of motion modify the period, dephasing the body from arriving at the intersection point simultaneously with the earth. An advantage of perturbations in this direction is that the miss distance grows with time so early action minimizes the necessary magnitude. As the orbit is still an intersecting one, the hazard is not eliminated for all time (so you cannot fire and forget). However, natural evolution is likely to the convert the orbit to a non-intersecting one before another collision possibility occurs. Because of these advantages, we consider develop 
an analytic relation capable of estimating the minimum speed change necessary to avoid an impact.

For an eccentric orbit, a fixed velocity change has its maximum effect when applied at perihelion. The quite general relation between the period change $(\delta P)$, and speed change $(\delta \mathrm{V})$. is:

$$
\frac{\delta P}{P}=3 \frac{\delta V}{V_{\text {aphelion }}} \text { at perihelion or } \frac{\delta P}{P}=3 \frac{\delta V}{V_{\text {perihelion }}} \text { at aphelion }
$$

or

$$
\frac{\delta P_{\text {perihelion }}}{\delta P_{\text {aphelion }}}=\frac{(1+e)}{(1-e)}
$$

Integrating over an extended period, the time at which a perturbed body crosses the earth's orbit has changed $(\delta \mathrm{t})$ by an amount:

$$
\delta t=t \delta P / P
$$

Where $t$ is the original time to impact. All that is required for a miss is that the time change permits the earth to move by its diameter, so that it is not at the impact position when the body passes the intersection point. Multiplying the earth's velocity $\left(\mathrm{V}_{\text {earth }}\right)$ which is relatively constant, by the time change provides an estimate of the miss distance:

$$
D_{\text {miss }}=V_{\text {earth }} \delta t=V_{\text {earth }} t^{\delta P} / P
$$

With this we can calculate the velocity perturbation necessary to stimulate a miss distance, $D_{\text {miss }}$, of $\mathrm{N}$ earth radii(for perihelion or aphelion):

$$
\begin{gathered}
\delta V_{\text {peri }}=\frac{N R_{\text {earth }}}{3 t V_{\text {earth }}}\left(\frac{G M_{\text {sun }}}{a_{\text {asteroid }}} \frac{1-e}{1+e}\right)^{1 / 2} \\
\delta V_{\text {aphelioni }}=\frac{N R_{\text {earth }}}{3 t V_{\text {earth }}}\left(\frac{G M_{\text {sun }}}{a_{\text {asteroid }}} \frac{1+e}{1-e}\right)^{1 / 2}
\end{gathered}
$$

As a test, these relations were compared to a precise numerical simulation. To do this, the equations were turned around, and used with a fixed speed change $(\delta \mathrm{V})$ to predict the miss distance $\left(\mathrm{NR}_{\text {earth }}\right)$ versus time $(\mathrm{t})$. The results are plotted on figure 5 , showing good correspondence. 
The direct prediction of these equations for a miss distance of one earth diameter ( 2 earth radii) is shown in figure 8 . As can be seen, a speed change of $10 \mathrm{~m} / \mathrm{s}$ secures a miss for an object less than a month out. Such an impulsive change is impossibly large for a body with little rigidity that has an escape velocity under a meter per second. However, if one were to shatter the body, this sets the scale for the velocity dispersion that will lead to material missing the earth.

Lower speed changes of $1 \mathrm{~m} / \mathrm{s}$ and $0.1 \mathrm{~m} / \mathrm{s}$ require reaching out to 100 or 1000 days (prior to impact) to ensure a miss. For this orbit, application of even $0.01 \mathrm{~m} / \mathrm{s}$ at perihelion can achieve a miss if applied a decade prior to impact.

Fig 8: The miss distance is shown for velocity perturbations applied along the direction of motion, between 10 and 10,000 days prior to impact. b) The minimum velocity that secures a miss for different times to impact

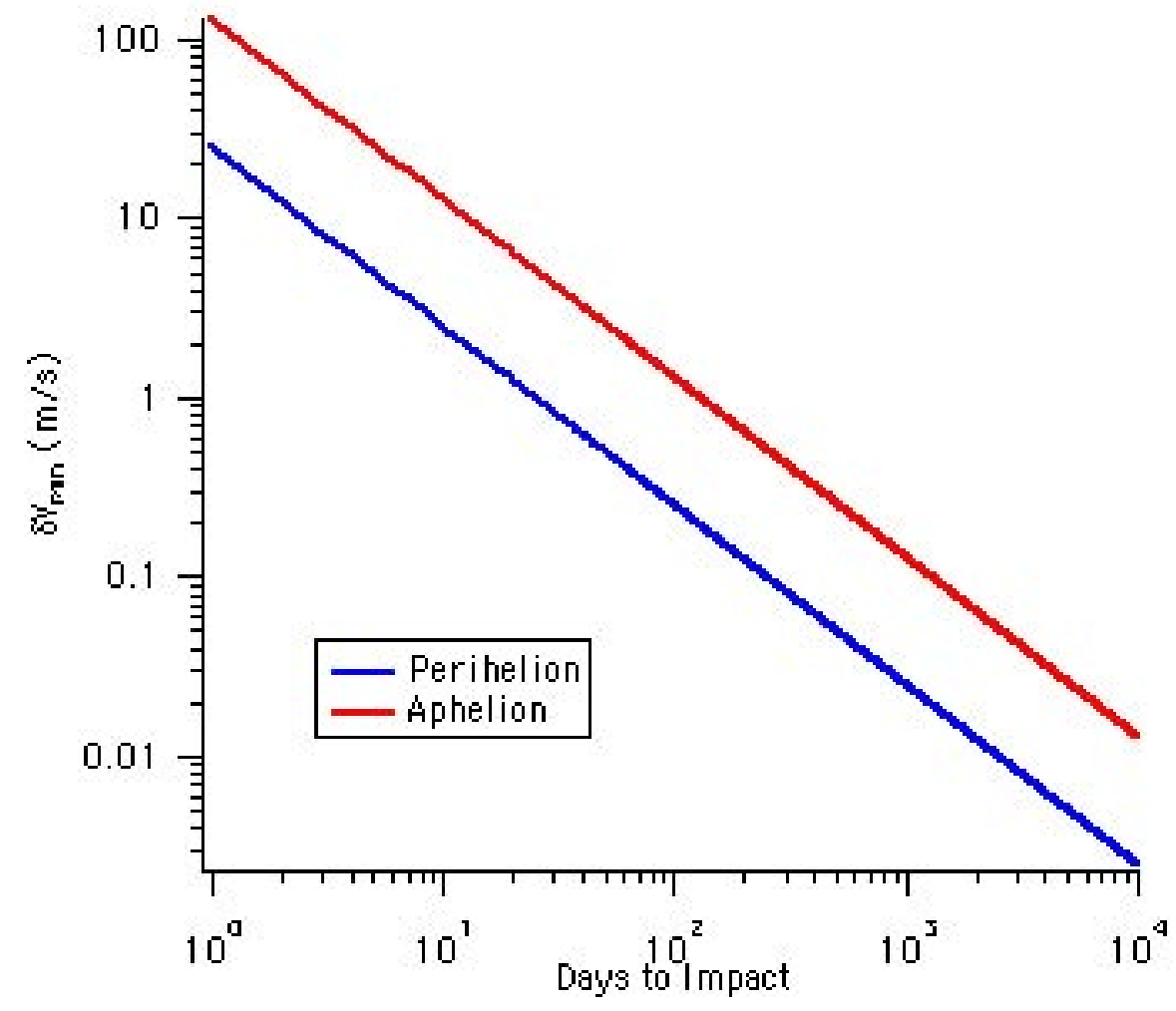

The examples that have been shown here were modeled for a particular NEO orbit, defined below as orbit 1 . To demonstrate generality, a range of orbits was considered for a quick, though non-exhaustive test for the conditions to divert an asteroid versus time. The orbital elements of these bodies are given in table 2 , and shown in figure 9 .

Table 2

$\begin{array}{clccc}\text { Orbit } & \mathrm{P} & \mathrm{a} & \mathrm{e} & \mathrm{i} \\ 1 & 0.6992 & 0.7878 & 0.67518 & 4.8650 \\ 2 & 1.2206 & 1.1422 & 0.50808 & 6.8238 \\ 3 & 1.3677 & 1.2322 & 0.35142 & 0.6149 \\ 4 & 3.5265 & 2.3169 & 0.65454 & 1.2101\end{array}$


Fig 9: the orbits of the earth (orange) as well as 4 typical NEO orbits are projected onto the ecliptic plane.

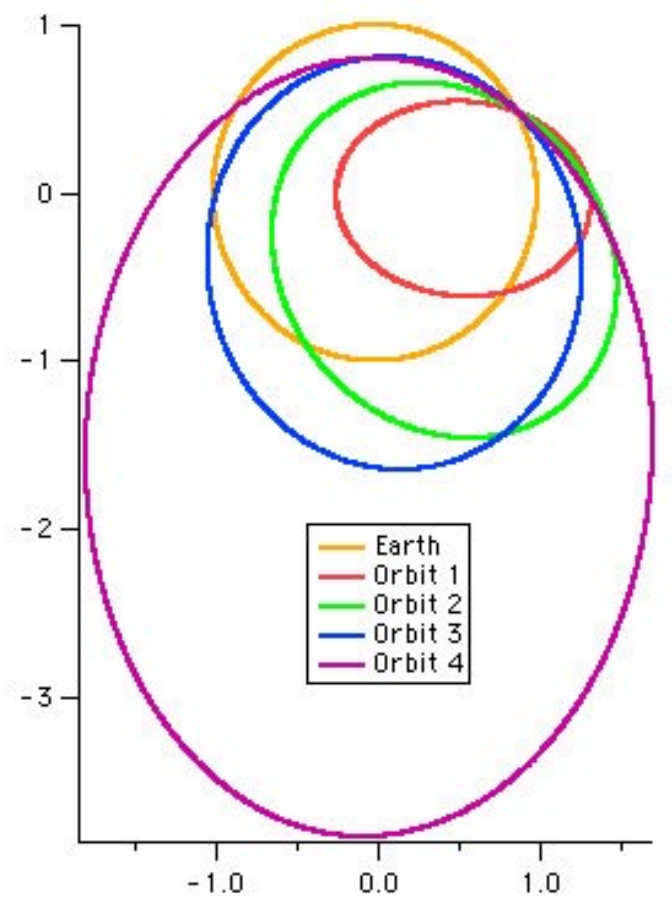

As is shown in figure 10, the aphelion miss distance for each orbit is well characterized by the analytic relations above. The same is true of miss distances for speed changes at perihelion.

Fig 10: The miss distances is shown for a speed change of $0.1 \mathrm{~m} / \mathrm{s}$ on various orbits. In all cases equation 1 provides a good estimate of the miss, shown here for aphelion impulses.

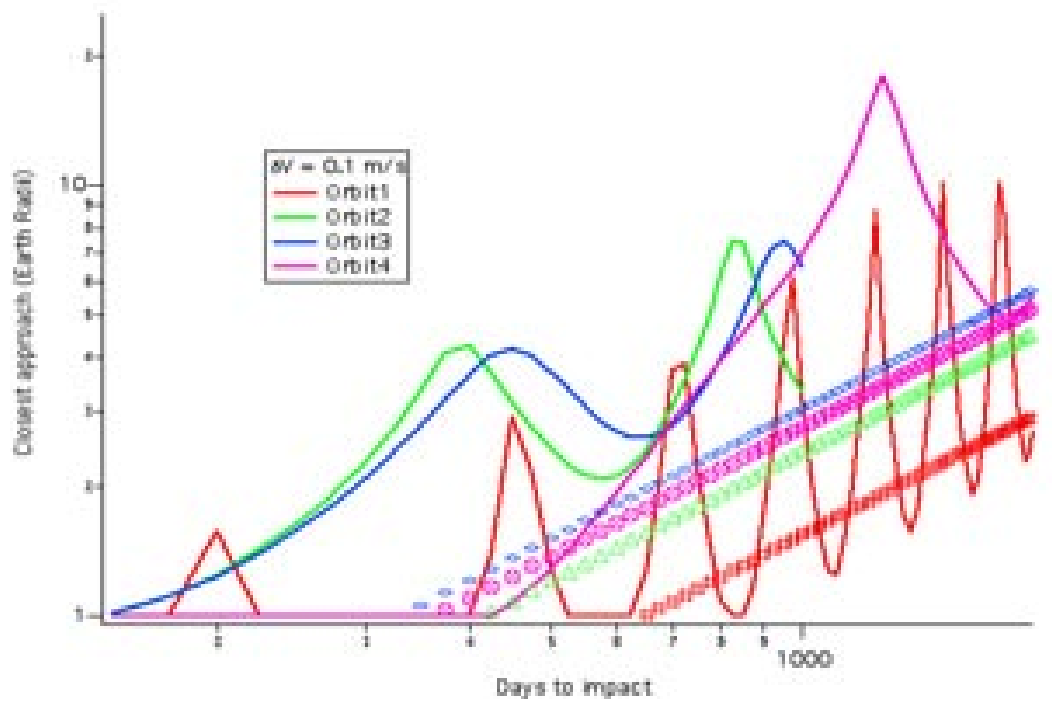


IM \#305615

\section{Minimum conditions for diverting an asteroid via continuous accelerations:}

As an alternative to a discrete impulse (or impulses), some mitigation strategies operate by application of a constant acceleration. Anchoring a thruster, and dealing with rotation as well as off-axis moments of inertia, are significant problems. The momentum necessary to de-spin a $1 \mathrm{~km}$. diameter object rotating with a 5-hour period is comparable with that necessary to speed it by $0.1 \mathrm{~m} / \mathrm{s}$. If the spin is not stopped, the rocket must operate in a high thrust mode for short periods. A high thrust mode exacerbates the difficulty of anchoring the thruster to the surface. Off-axis moments of inertia will also be particularly troublesome if the rotation is allowed to continue. Nevertheless, these are issues that are being examined an might be solved, so for a first glance on the required thruster technology, we will give no regard to these difficulties.

As for the impulsive velocity change case, an acceleration aligned with the direction of motion produces the maximum effect. Numerical simulations show that for times to impact less than a single orbit, the acceleration required, a, shows a substantial sensitivity to the to the orbital phase. As expected this sensitivity is considerably smoothed when the acceleration is applied more than an orbital period prior to impact. For the standard orbit used above (orbit 1 ), the minimum acceleration, $a_{c c}$, necessary to deflect the body for a miss distance of less that 0.01 earth radii (a bit more than the orbit of the International Space Station) was determined numerically for a $10^{12} \mathrm{~kg}$ body. If we take, $V_{\text {exhaust }}=2.1 \mathrm{~km} / \mathrm{s}$ (appropriate for a kerosene and hydrogen peroxide mixture), we find the results shown in figure 11.

Fig 11 The thrust (lbs) and fuel mass (tons) for the constant acceleration necessary to secure the miss of a $10^{15} \mathrm{gm}$ object
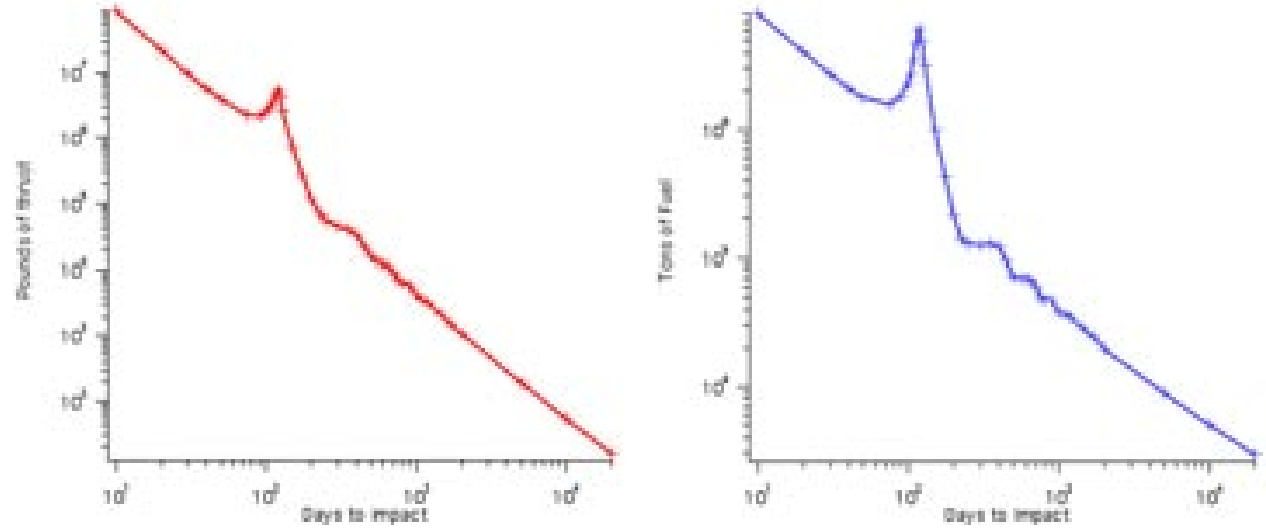

The acceleration was assumed to be constant, and applied over the entire time to impact $(\mathrm{t})$. The thrust necessary to produce this acceleration is:

$$
\text { Thrust }=M_{\text {asteroid }} a_{c c}
$$

The mass of fuel necessary to cause this acceleration (assuming that $V_{\text {exhaust }}>a_{c c} t$ ) is:

$$
M_{\text {fuel }}=M_{\text {asteroid }} a_{c c} t / V_{\text {exhaust }}
$$


As expected, attempting to accelerate the asteroid in its final 10 days of approach to the earth requires horrendous thrust (above that of the Saturn 5 booster), with a minimum of 8 million tons of fuel (immutability and Newton). As expected, there is a substantial reduction of the reaction mass required if one can land sufficient mass on the body at an early time.

If the acceleration is begun 20,000 days prior to impact (55 years), only about 16 lbs of thrust are required, but the total fuel bill is still substantial, about 3,000 tons of reaction mass. Changing the reactant mixture to liquid hydrogen and oxygen (and neglecting the long term storage difficulties associated with handling hydrogen) could reduce the reactant mass to about 1000 tons, but still requires a 55 year burn.

While ion thrusters are still a developmental technology, their exhaust velocity has the potential to reduce the reaction mass problem considerably. The current NASA effort, DS1, uses Xenon as the propellant, and accelerates it to about $30 \mathrm{~km} / \mathrm{s}$. This would reduce the reaction mass requirement to only 200 tons, but there is a substantial requirement for electrical generation. An ionic thruster uses electrical energy, not only to provide kinetic energy to the ion, but to generate and manipulate the ion, as well as produce an electron beam to neutralize the exhaust material. DS1 uses about $2.5 \mathrm{Kw}$ to produce a thrust of 92 milli-Newtons, or about $1 / 50^{\text {th }}$ of a pound. Scaling from this, a $16 \mathrm{lb}$ thruster requires a continuous generation rate of about 6 megawatts for 55 years (for a $10^{12} \mathrm{~kg}$ body). If the body has a small semi-major axis (spent most of its time close to the sun), and they lost no efficiency with time, this energy could be produced by about 4 acres (1.7 hectares) of solar panels kept continuously in the sun. In the presence of rotation, continuous generation is most simply achieved by placing 4 such solar energy farms around the asteroid. Alternatively, a small nuclear reactor (with minimal shielding for un-manned operation) might be designed to accommodate the power requirements. In either case, there will be a non-negligible weight cost.

Following the analysis of how an impulsive velocity change integrates to a miss distance, an analytic relation for the minimum acceleration can be developed. Begin by defining an effective velocity perturbation, $\delta \mathrm{V}_{\text {eff }}$.

$$
\delta V_{e f f}=a_{c c} t / 2
$$

The average velocity for an orbiting body with an eccentric orbit is:

$$
V_{\text {avg }} \approx \frac{2 \pi a\left(1-e^{2} / 2\right)^{1 / 2}}{P}=29.5 \mathrm{~km} / \mathrm{s}
$$

Using this average velocity provides a relation between the velocity perturbation and the accumulated miss distance:

$$
D_{\text {miss }}=V_{\text {earth }} \delta P / P=\frac{3 t V_{\text {earth }} \delta V_{\text {eff }}}{V_{\text {avg }}}=3 / 2 a_{c c} t^{2}\left(V_{\text {earth }} / V_{\text {avg }}\right)
$$


Avoiding an impact requires a miss distance greater than 2 earth radii. For times greater than a couple of orbits (about 510 days in this case) this equation can be used to estimate the minimum acceleration that secures a miss:

$$
a_{\text {miss }}=4 / 3 \frac{R_{\text {earth }}}{t^{2}}\left(V_{\text {earth }} / V_{\text {avg }}\right)
$$

In figure 12, we present the ratio of the numerically determined acceleration resulting in a miss, $a_{c c}$, to the estimate determined here, $a_{\text {miss. }}$. Over two orders of magnitude range in time (from 200 days to 20,000 days) our simple analytic approach yields a reasonable estimate of the minimum acceleration necessary to secure a miss. For times shorter than 200 days, the acceleration is applied for only a fraction of an orbit, and the result is very sensitive to the precise start time.

Fig 12: Ratio of the numerically determined acceleration to the estimate

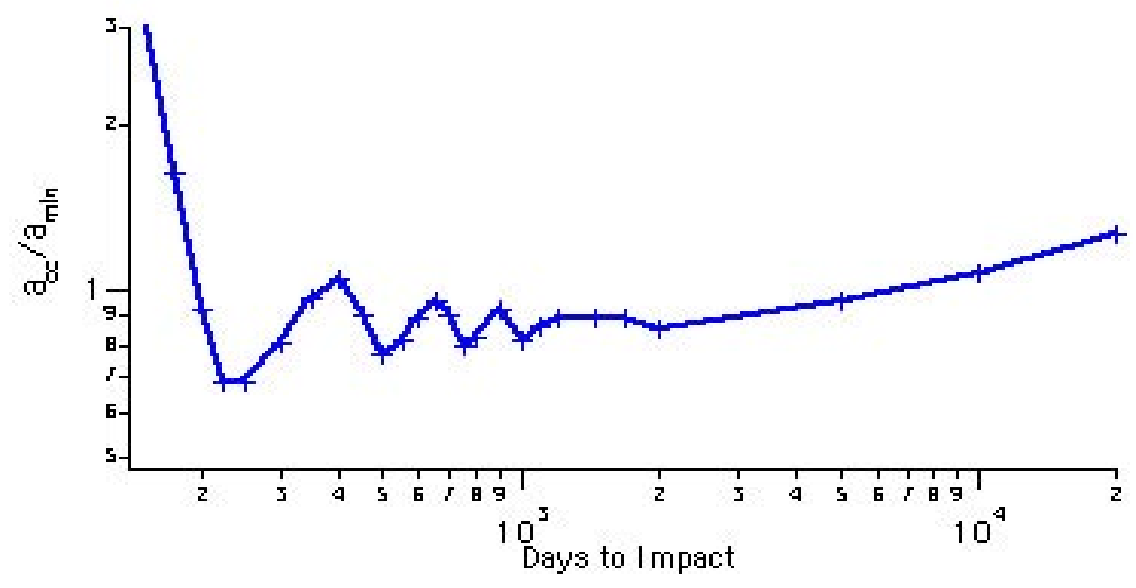

Of course, the accelerations determined here resulted in a miss of 0.01 earth radii (or less), and a somewhat larger margin would be desirable. Beyond the rotation and anchoring problems mentioned earlier, there are many technical challenges associated such long-term continuous operation, or the lifting this much mass, setting it gently on the asteroid, and maintaining it there. Nevertheless, some type of "rocket" technology might be developed when there are centuries of advanced notice. This is particularly true of ion thruster technologies where technological improvements are likely to be quantitatively larger.

\section{Shattering the asteroid, or the Hollywood approach:}

On the earth's surface (in dry soil), a one-megaton device digs a crater that is about 150 meters in radius and 75 meters deep, ejecting approximately $3.5 \times 10^{9} \mathrm{~kg}$ of material. On or a bit below the surface, the intense radiation field burns outward at a speed greater than the shock speed, ionizing all surrounding material and resulting in a strong shock as the high-pressure plasma bubble expands. The energy density in this fireball exceeds that of high explosive, and the expansion stresses nearby rock beyond its elastic limits resulting in a region of fracture and melt. Even unconsolidated alluvial material is strongly accelerated as seen on 6 July 1962, when the Sedan test was done 
as part of the LLNL Plowshare program. This nuclear excavation experiment created a crater $390 \mathrm{~m}$ in diameter and 100 meters deep. It did this by using a 104kiloton device to lift about $10^{10} \mathrm{~kg}$ sufficiently fast to move it hundreds of meters in the earth's gravity $(>45 \mathrm{~m} / \mathrm{s})$. Showing that burial improves the energy coupling and results in a much larger crater from a smaller yield device

To lift this mass, and throw it to the side, utilized at least $3 \%$ of the available energy. This small utilization was an intentional choice to assure (most of) the bomb debris would fall back into the crater, and be well buried. The result is that an amount of mass comparable to that of a 200 meter asteroid, was impulsively accelerated to many 10's of meters per second, using a nuclear device with a mass well under a ton.

Fig 13: the formation of the Sedan Crater on 6 July 1962.

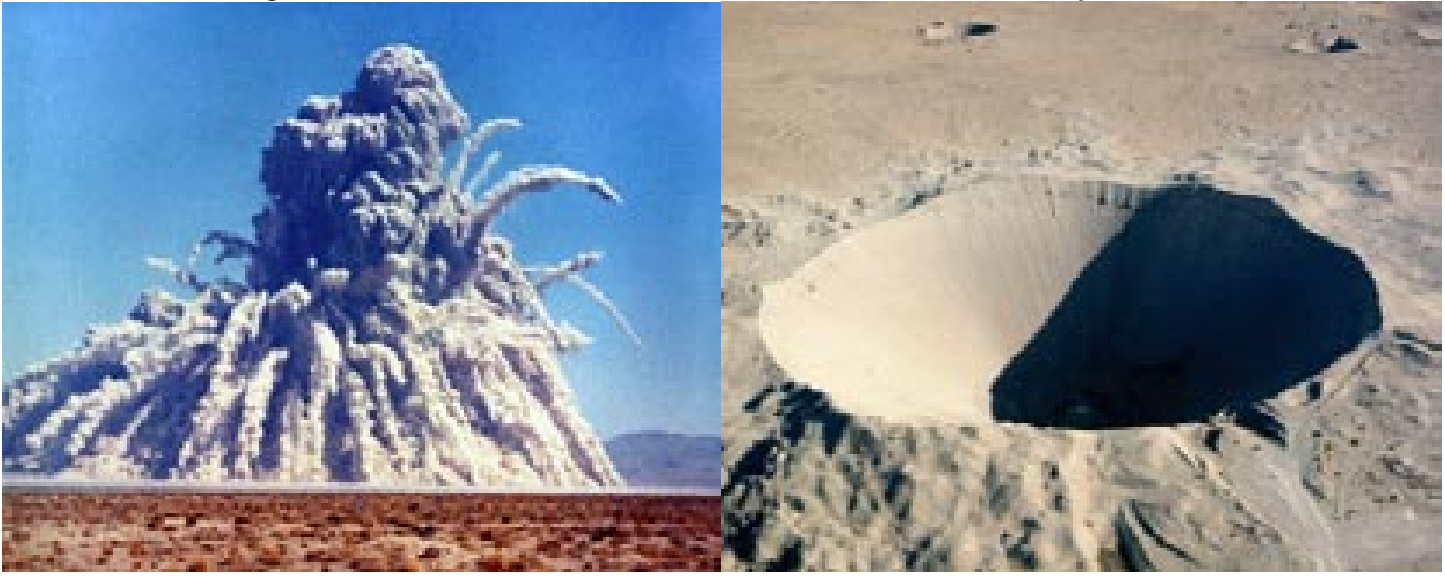

As shown in the discussion on impulsive velocity perturbations, speeds of this magnitude enable pieces of the asteroid to follow a path where they entirely miss the earth even if applied only a few days prior to impact. Additionally, the simulations presented above, showing miss distances for impulses in the directions orthogonal to the motion, also show that velocities of $10 \mathrm{~m} / \mathrm{s}$ result in misses on a short timescale. The two fundamental questions for evaluating the efficacy of shattering an asteroid as a defense mechanism are the coupling efficiency, and the evolution of the debris ellipsoid.

Fig 14: The RMS velocity from 0.1 megatons coupled into an object of density $5 \mathrm{~g} / \mathrm{cc}$, including gravitational binding energy.

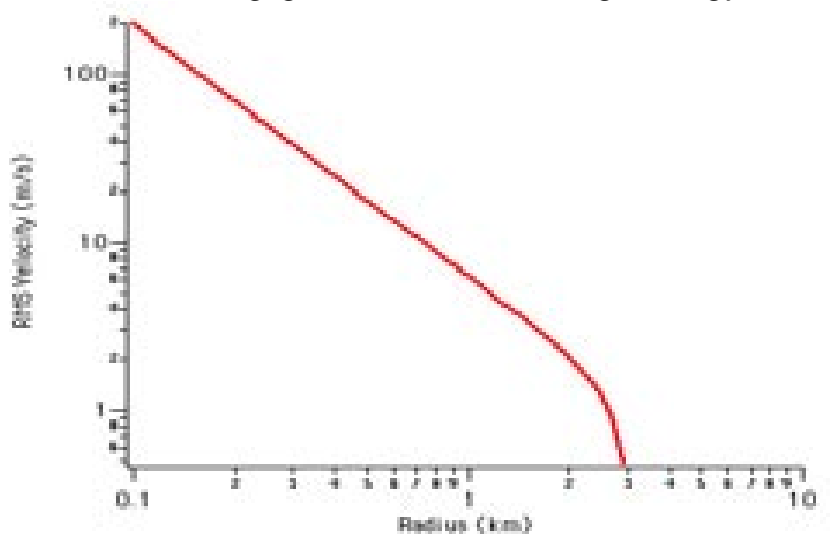


The Sedan crater was large, in spite of the inefficient depth of burial, because the medium had little mechanical strength. However, current consensus suggests that many large asteroids may also be very friable, or is in essence a weakly bound gathering of debris. As a crude estimate of what might be done, a megaton device converting $10 \%$ of the energy (less the gravitational potential energy) to motion into a $500 \mathrm{~m}$ in radius body produces a velocity distribution with an RMS value near $20 \mathrm{~m} / \mathrm{s}$. Smaller bodies require even less coupling to produce velocity distributions of interest.

Returning to the four NEO orbits considered above, consider a 400 meter diameter body with a density of $2 \mathrm{~g} / \mathrm{cm}^{3}$ and a mass near $6.7 \times 10^{10} \mathrm{~kg}$. If only $0.5 \%$ of the energy of a 1 MT explosive can be coupled into the material, it will have an RMS velocity of $25 \mathrm{~m} / \mathrm{s}$. Assuming that the material is ejected equally in all directions, the debris will form an ellipsoidal shape, through which the earth will tunnel (table 3 ).

For each orbit, the destructive impulse was applied at the perihelion passage nearest 1000 days before impact. Over the roughly 3 years to impact, the debris ellipsoids grew to such a size that less than $2.4 \times 10^{-4}$ of the material remained on a collision path. The energy of the material that still impacts the earth is reduced from thousands of megatons, to under a megaton, and is spread over a distance that it requires nearly an hour for the earth to transverse (52 to 85 minutes) it. If this dispersed material is composed of small debris that burns up in the atmosphere, the additional thermal loading of the earth is less than $1 / 100,000^{\text {th }}$ of the constant solar input (43 megatons/second). If all of this material is concentrated in a single piece, its equivalent diameter is reduced to under 24 meters. Pieces this small can be mitigated with additional application of force in the final days, or at the very worst are a much more localized problem.

Table 3

$\begin{array}{ccccc}\text { orbit } & \begin{array}{c}\text { Impact } \\ \text { energy } \\ \text { Megatons }\end{array} & \begin{array}{c}\text { Intercepted } \\ \text { Fraction }\end{array} & \begin{array}{c}\text { Actual } \\ \text { Energy } \\ \text { Kilotons }\end{array} & \begin{array}{c}\text { Duration } \\ \text { of Impacts } \\ \text { Minutes }\end{array} \\ 1 & 4200 & 2.3 \times 10^{-4} & 960 & 52 \\ 2 & 3200 & 4.0 \times 10^{-5} & 128 & 80 \\ 3 & 1800 & 3.3 \times 10^{-5} & 59 & 62 \\ 4 & 2900 & 7.8 \times 10^{-6} & 37 & 85\end{array}$

Clearly, the result of sweeping through the debris of a dispersed body is a tremendous improvement over letting nature take its course, but there is a statistical element to this approach that is not reassuring. Nevertheless, direct dispersal is a positive option for dealing with small bodies when there is very limited time.

The small size is also helpful in achieving, the assumed condition that material is dispersed equally in all directions. This requires emplacement of the explosive in the center of the object, or at least very deeply. If the surface is truly unconsolidated rubble, Earth-penetrator technology could easily deliver the device many 10's of meters below the surface with a very modest weight penalty. 
Before leaving this approach, the Portho's case posed by this conference presents special problems. It is a large (kilometer scale) long period comet in a high inclination orbit that will approach the impact point from the southeast striking with a speed of 62 $\mathrm{km} / \mathrm{s}$. The object is a classical "dirty snowball" and rotates once every four days. As is true of such objects, discovery is seldom more than 1000 days before the arrival in the inner solar system. In the absence of any mitigation, the impact energy would be in excess of 45 million megatons, a global catastrophe.

Using a Monte Carlo code operating on a parallel machine, it was straight-forward to study the result of an explosive dispersal 260 days. Following nearly 40,000 pieces that were dispersed with an RMS velocity of $20 \mathrm{~m} / \mathrm{s}$, resulted in a debris ellipsoid of size $(1 \sigma)$ :

\begin{tabular}{ccc} 
direction & \multicolumn{2}{c}{ Standard Deviation } \\
$\mathrm{T}$ & 78.74 & Earth radii \\
$\mathrm{N}$ & 40.24 & Earth radii \\
$\mathrm{W}$ & 22.57 & Earth radii
\end{tabular}

With this dispersal, the earth impacts only about $10^{-4}$ of the total body. While this is still some thousands of Megatons of impact energy, it is spread over hours, and is again a considerable improvement over allowing nature to take its course. Dispersal could be the difference between a civilization-ending event, and serious regional rebuilding task. However, to intercept Porthos 260 days before impact requires delivering a ton scale package nearly 3.5 AU from earth. Achieving that in the roughly 700 days between discovery and the detonation time would not allow time for anything but reaction (a come as you are event).

In conclusion, when there are relatively short times to impact, a few years, asteroid disruption can reduce the expected impact damage considerably. Sufficiently small objects could be almost entirely mitigated, and the debris clouds might be observed to detect any remnants large enough to be of additional concern. If a piece as large as 100 meters is fragmented and spread evenly over a $3000 \mathrm{~km}$ square (requiring a dispersal of $20 \mathrm{~m} / \mathrm{s}$ applied at about 5 times the distance of the moon), the thermal loading will not exceed $1 \mathrm{cal} / \mathrm{cm}^{2}$, insufficient to cause damage. The time dispersal of the arriving debris will further lower the thermal loading problem.

Because this approach provides only statistical safety, not assured safety, it is not the preferred method unless time is short. If time is sufficient, a more controlled approach as described below will generally be more satisfying.

\section{Small Impulsive Perturbations, or the Nuclear Nudge}

As discussed above, a small fraction of a megaton is sufficient to disperse a $1 \mathrm{~km}$ scale body with 10's of meters per second. An even smaller fraction of that nuclear energy is necessary for velocity perturbations of $0.1 \mathrm{~m} / \mathrm{s}$, yet it was shown that speed changes in the range 0.01 to $0.1 \mathrm{~m} / \mathrm{s}$ are sufficient to divert an asteroid with a few years to decades of advanced notice. The question is how to apply such a nudge to a body whose structural integrity is uncertain. Structural rigidity is a major unknown for 
asteroids in general, and is likely to be variable. The lack of a short rotation period in any object larger than 200 meters argues that kilometer scale objects are composed of $10^{6}$ or more, smaller pieces.

Without experimental data of the sort that would have been gathered by the cancelled Clementine II mission, the only binding of which we can be confident is gravitational. In figure 15, we see that even very small objects of a few hundred meters diameter have escape velocities above $0.1 \mathrm{~m} / \mathrm{s}$, and might have difficulty surviving an impulse to mor than a couple of centimeters per second. As discussed above, these objects may be dealt with explosively. By the time the object diameter has increased to $1 \mathrm{~km}$, the escape velocity is of order $0.5 \mathrm{~m} / \mathrm{s}$, and for very large bodies $(>10 \mathrm{~km})$, the escape velocity approaches $10 \mathrm{~m} / \mathrm{s}$. If the explosive energy is coupled into a broad area of surface to give an even push across a hemisphere, it should be possible to avoid velocity dispersions larger than the escape velocity, and the bulk of the material will remain gravitationally bound. Material strength will only improve this result (Solem,2000).

Fig 15: Escape velocity Vs. Radius for different density objects.

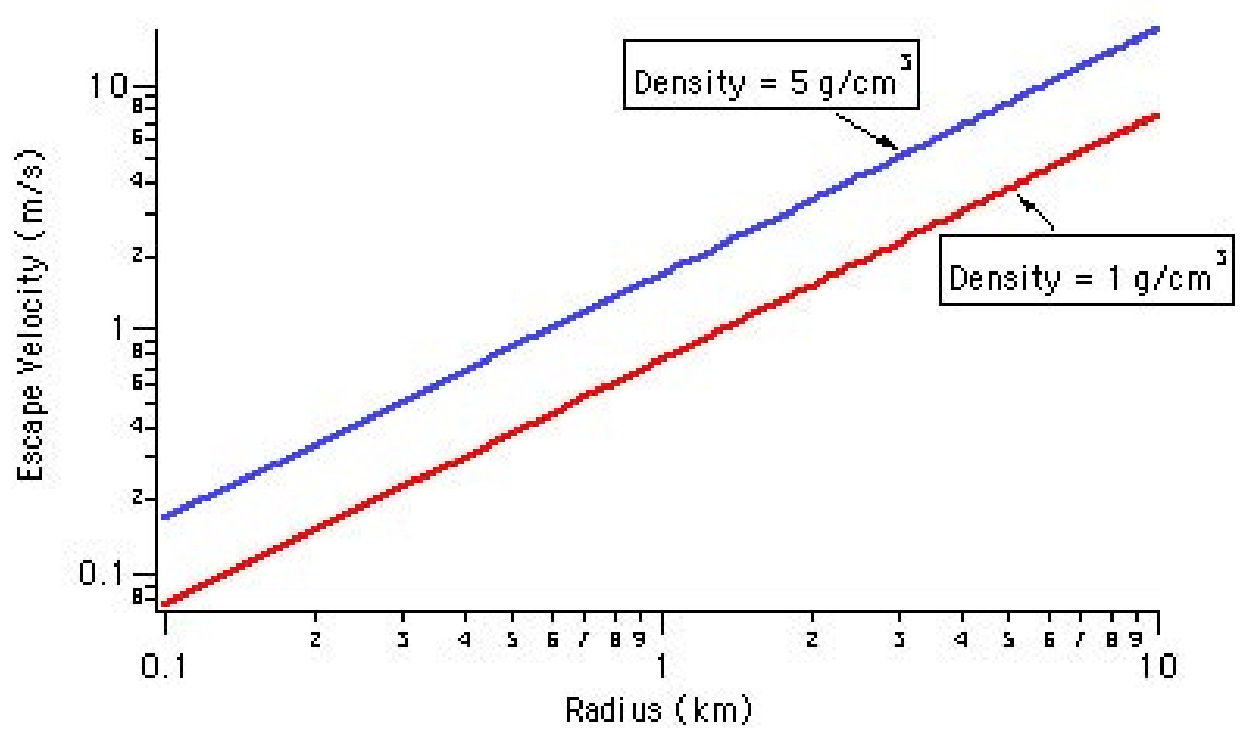

As described in Glasstone and Dolan (1977), most of the energy leaves a nuclear device in the form of radiation. In the atmosphere, that energy couples into the surrounding air, forming the fireball that expands producing a blast effect. In space, there is no air, so the radiation interacts directly with the surface of the asteroid.

Because nuclear explosives contain such a high energy density, an alternate method of deployment is possible in which the flux is diluted by detonation above the body. Distance not only dilutes the radiative flux, but results in a gentler impulse over a broader area. The deployment also eliminates the necessity of a controlled landing. The lower temperature, and wide spread impulse greatly reduces the high velocity gradients associated with a surface burst. 
As an example of such deployment, consider detonating a device above the body's surface (figures 16 and 17). Here, the fraction of the energy used depends on the solid angle subtended by the asteroid. Detonation at lower altitude will use more of the energy, but will expose a smaller section of the body to the energy, and result in stronger gradients.

Fig 16: The irradiated region of from a nuclear detonation one asteroid radius above the

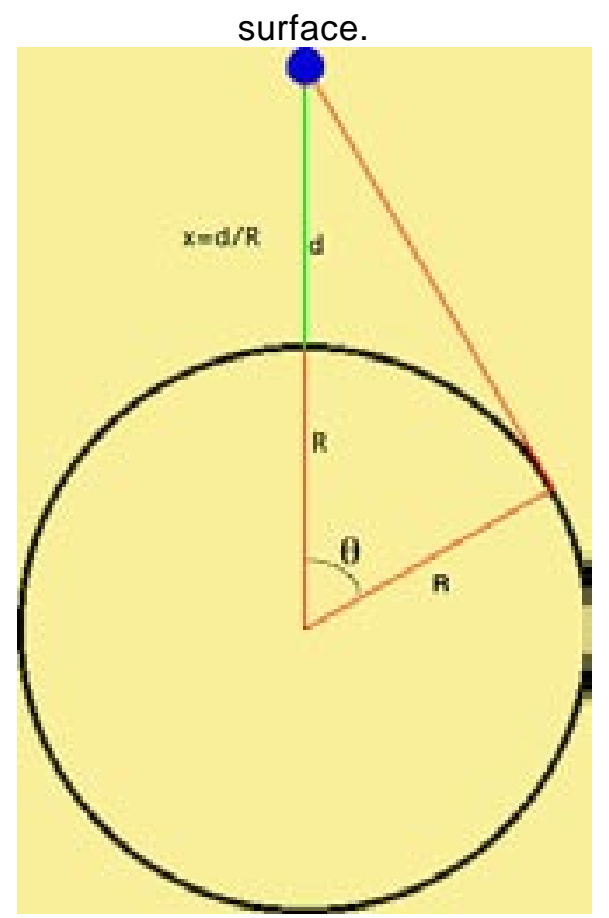

Fig 17: Fraction of the yield that intercepted by the asteroid versus height of burst.

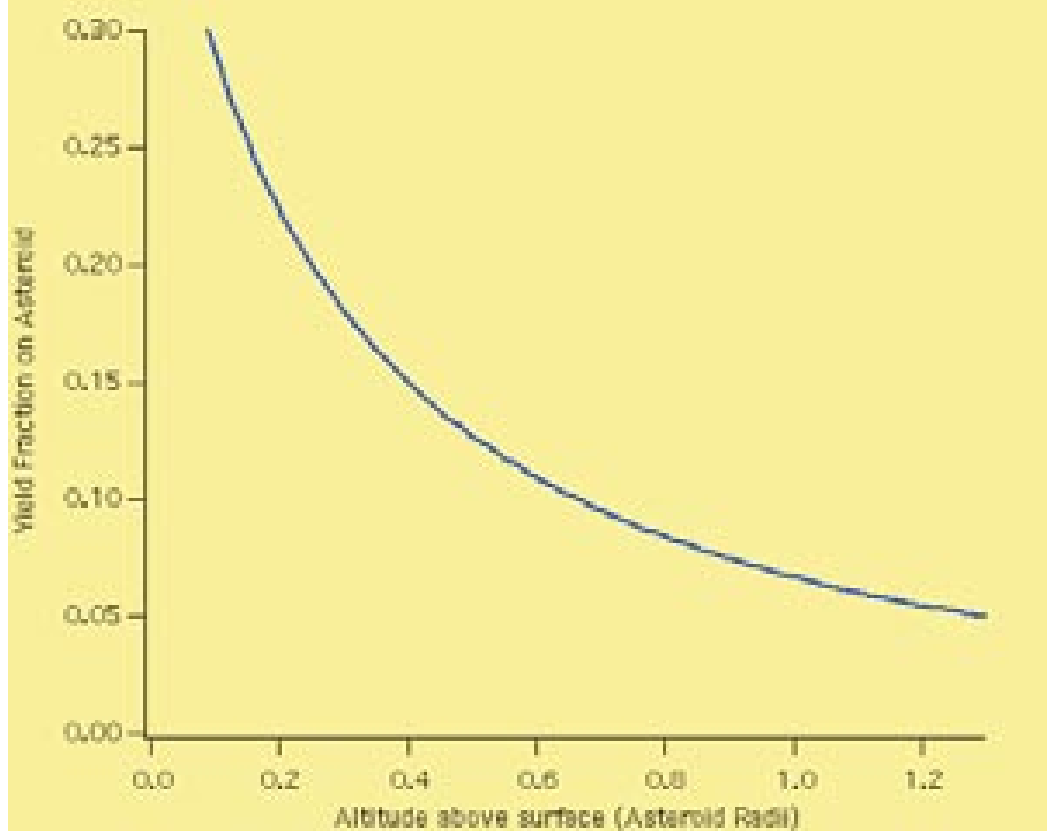


The maximum heating is experienced at the point nearest the detonation (ground zero). As one moves away from the ground-zero point, the flux received from the device is reduced as by distance, and the asteroid curvature causes the energy to be spread over more surface area. If the height of burst, $d$, is parameterized in terms of the asteroid radius:

$$
x=d / R
$$

The flux distribution across the irradiated region is figure 18):

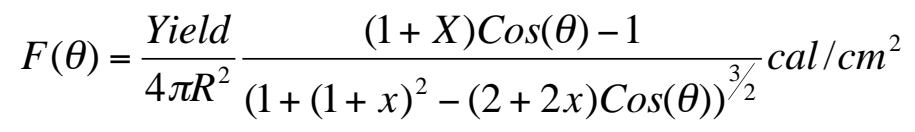

Fig 18: The flux distribution versus angle form the ground zero point for a $1 \mathrm{~km}$ asteroid. The yields were adjusted to provide the same fluence at ground zero.

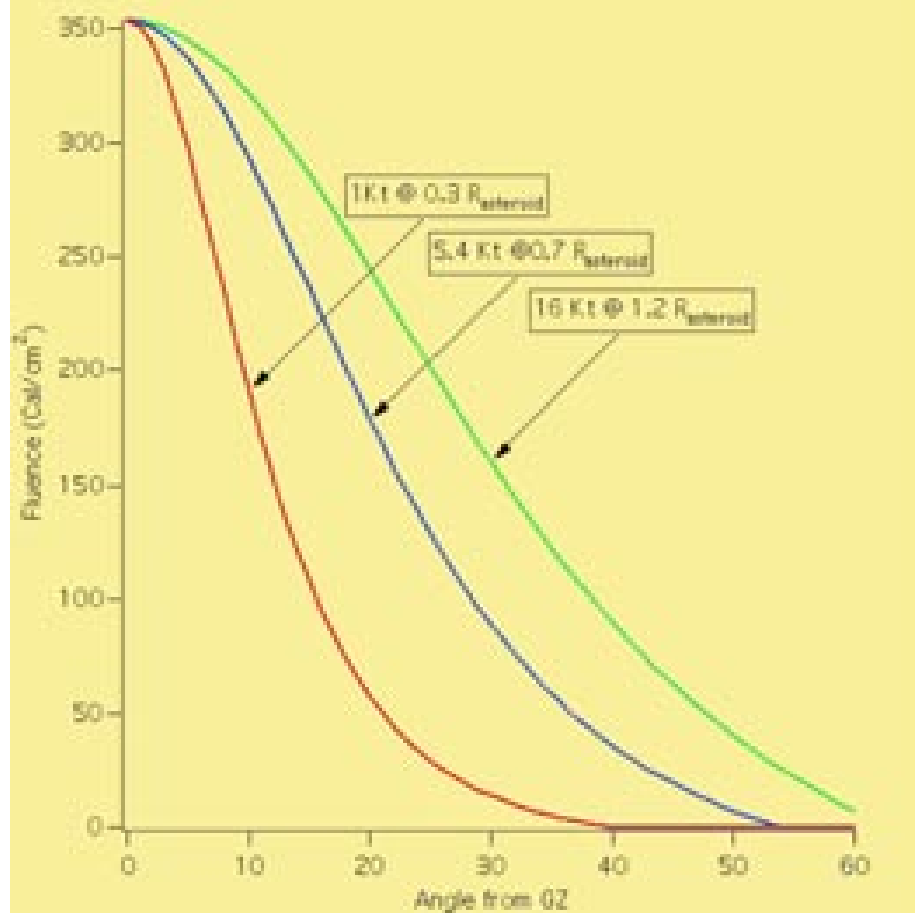

The response of the surface to heating depends on the type of radiation for which the nuclear device optimized (soft X-rays, hard X-rays, $\gamma$-rays, neutrons, $\ldots$ ), as well as the surface composition. Starting with the surface composition issue, if we take the body to be composed of olivine (a mixture of forsterite and fayalite), raising the temperature to about $1300 \mathrm{C}$ (requiring about500 cal/gm) will result in a cheery glow, but little else. While this might be used to provide a fusion crust on a weak surface, it does little to add impulse to the body.

With the latent heat of fusion, another 2000 calories per gram will vaporize the surface material, and raise the temperature to about $1 \mathrm{ev}$, with a sound speed of about 2.4 $\mathrm{km} / \mathrm{s}$. In this case, a surface layer of the facing hemisphere has been given an energy content comparable to a chemical explosive or rocket fuel. This thin vaporized layer will expand at an average velocity $\left(\mathrm{V}_{\text {ejecta }}\right)$ comparable to the sound speed. 
This weak shock, generated by the gas expansion, propagates through the bulk of the asteroid resulting in a momentum change equal to that of the ejected surface layer. A heavily rubblized body should be particularly dissipative, via crushing and surface shearing, spreading the energy evenly through its mass, reducing possible velocity gradients. The magnitude of the asteroids velocity change, $\delta \mathrm{V}$, then depends on the mass that is ejected, $\delta \mathrm{M}_{\text {ejecta }}$ :

$$
\frac{\delta M_{\text {ejecta }}}{M_{\text {asteroid }}}=\frac{\delta V}{V_{\text {ejecta }}}
$$

The ejecta mass depends on the fraction of the surface that is irradiated, and by how deeply you couple energy into the surface material. As described in the introduction, the mass ejection at speeds of order $1 \mathrm{~km} / \mathrm{s}$ requires 10's to 100's of thousands of tons to accelerate a $1 \mathrm{~km}$ object by centimeters per second. Assuming the penetration depth to decrease with the cosine of the incident angle, the depth of penetration necessary to eject a chosen fraction of mass (and so to a chosen speed) is:

$$
N \lambda=2 R \delta M_{\text {ejecta }} / 3 M_{\text {asteroid }} \int_{0}^{\theta_{\max }} \frac{[(1+x) \operatorname{Cos}(\theta)-1] \operatorname{Sin}(\theta) d \theta}{\left[1+(1+x)^{2}-2(1+x) \operatorname{Cos}(\theta)\right]^{1 / 2}}
$$

As shown in figure19, bursts at a low altitude (small $x$ ) must penetrate very deeply to heat sufficient mass. Raising the burst altitude increases the area exposed to the explosion energy, and reduces the required penetration depth.

Fig 19: The relation between the penetration depth $(N \lambda)$ and height of burst to change the velocity of a $1 \mathrm{~km}$, density $2 \mathrm{~g} / \mathrm{cm}^{3}$ asteroid by $0.01 \mathrm{~m} / \mathrm{s}$. A $0.1 \mathrm{~m} / \mathrm{s}$ change requires ten times the mass and so ten times the penetration depth.

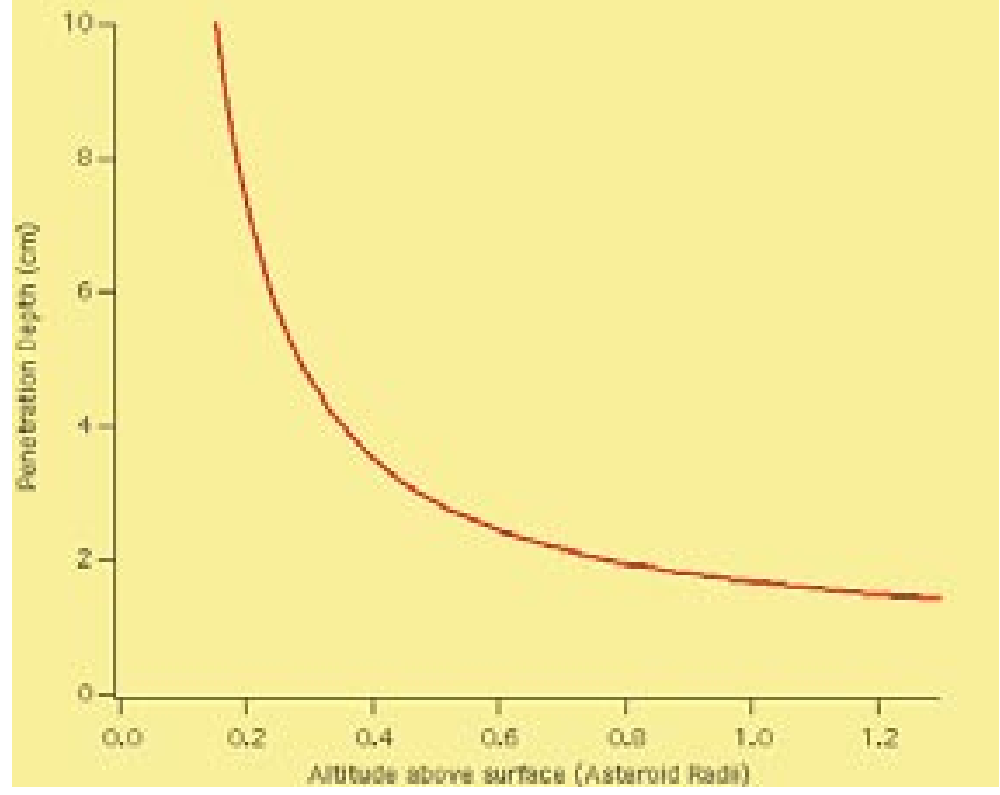


Perturbing the speed of a kilometer scale asteroid by 1 to $10 \mathrm{~cm} / \mathrm{s}$ requires 2 to $20 \mathrm{~cm}$ of penetration, or more generally, penetration to a column density of 4 to $40 \mathrm{~g} / \mathrm{cm}^{2}$. The X-ray mean free path into cold silicates is rather short (10's of microns), as X-rays of $1 \mathrm{Kev}$ penetrate in only about $10^{-3} \mathrm{~cm}$. Even harder $(10 \mathrm{Kev}) \mathrm{X}$-rays have a mean free path of only $10^{-1} \mathrm{~cm}$. While X-rays can burn into the asteroid (as the surface layer ionized, the mean free path increases allowing more penetration), this approach results in higher temperature surface gas, and sharper pressure pulses. Alas, as the temperature increases, re-radiation (effectively albedo) considerably reduces the efficiency of coupling energy into the asteroid. Finally, of the types of output mentioned above, X-rays are more sensitive to precise elemental abundances than $\gamma$ rays or neutrons.

A better match to the penetration depths required for a single impulse is obtained by using multi-Mev neutrons. Devices that are efficient in producing such neutrons were developed in the plowshare program, and neutrons naturally have a mean free path in the centimeters range. Above $2 \mathrm{Mev}$, the neutron cross-section on oxygen, silicon, and magnesium is about 2 barns, independent of energy and about 3 barns for iron. The resulting mean free path is in an olivine mixture is:

\section{$\lambda=16.6 / \rho \approx 6.4 \mathrm{~cm}$}

To test this, a three dimensional Monte Carlo simulation was done (by Peter Bedrossian of LLNL) to find the fraction of the incident neutron energy that coupled into a surface. A composition similar to granite and had a density of $2.6 \mathrm{~g} / \mathrm{cm}^{3}$. All known reactions $\left[\left(n, n^{\prime}\right),(n, g),(n, a),(n, n p),(n, n a), \ldots\right]$ were includes. The result shows that for energetic neutrons of all energies, approximately $70 \%$ of the incident energy couples into the surface, and even with the high scattering cross sections and shallow penetration angles, less than $1 / 3^{\text {rds }}$ of the energy escapes. For neutrons above $2 \mathrm{Mev}$, over half of the deposited energy ( $40 \%$ of the total) couples into the first 10 centimeters or $\left(26 \mathrm{gm} / \mathrm{cm}^{2}\right)$, and nearly $85 \%$ of the deposited energy $(60 \%$ of the total) is coupled into the top 17 centimeters (or $44 \mathrm{~g} / \mathrm{cm}^{2}$ ). Because of enhanced capture cross-sections, lower energy neutrons $(1 \mathrm{Mev})$ deposit their energy into the top 10 centimeters. These penetration depths are well suited to the requirements for described above. While the penetration depths (in centimeters) listed here are density dependant, the more important number for our purpose, the column depth $\left(N \lambda \rho \mathrm{g} / \mathrm{cm}^{2}\right)$, is not. The ejecta mass is directly proportional to the column depth, and so neutrons will penetrate to the depth necessary to heat the requisite ejecta mass:

$$
\delta M_{\text {ejecta }}=2 \pi R^{2} N \lambda \rho \int_{0}^{\theta_{\text {max }}} \frac{[(1+x) \operatorname{Cos}(\theta)-1] \operatorname{Sin}(\theta) d \theta}{\left[1+(1+x)^{2}-2(1+x) \operatorname{Cos}(\theta)\right]^{1 / 2}}
$$

The flux profile, coupled with the albedo, density, and (cosine tapered) penetration depth, results in an energy per gram deposition. Dividing this by the specific heat results in a temperature profile: 


$$
\delta T(\theta)=\frac{\delta T_{\max }}{1+(1+x)^{2}-(2+2 x) \operatorname{Cos}(\theta)} \operatorname{deg} \text { rees }
$$

where

$$
\delta T_{\max }=\frac{\text { Yield }}{4 \pi R^{2} N \lambda \rho C_{p}} \text { deg rees }
$$

Fig 20: $0.95 \mathrm{Mt}$ of total neutron output detonated 300 meters above a $1 \mathrm{~km}$ body of density 2 body, results in the following temperature profile.

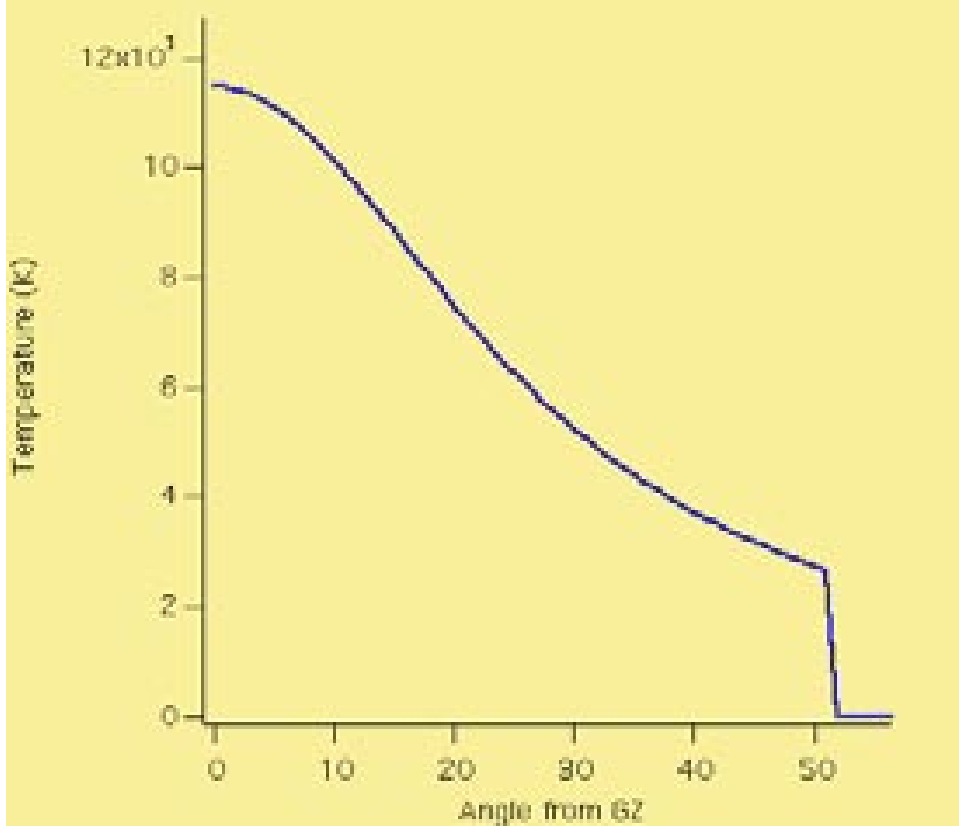

As shown in figure 20 the reduction in fluence near the explosion horizon is partially compensated for by reduced penetration of depth. This results in an impulse that is stronger near ground zero, and tapers to the limb.

If we assume that the heated material is locally ejected with the sound speed, the impulse $(\mathrm{g} \mathrm{cm} / \mathrm{s})$ is:

$$
I(\theta)=2 \pi R^{2} N \lambda \rho c_{s} \frac{(1+x) \operatorname{Cos}^{2}(\theta)-\operatorname{Cos}(\theta)}{\left(1-(2+2 x) \operatorname{Cos}(\theta)+(1+x)^{2}\right)^{1 / 2}}=2 \pi R^{2} N \lambda \rho v \delta T_{\max }^{1 / 2} \frac{(1+x) \operatorname{Cos}^{2}(\theta)-\operatorname{Cos}(\theta)}{1-(2+2 x) \operatorname{Cos}(\theta)+(1+x)^{2}}
$$

Considering only the component of the impulse that is parallel to the direction of push (the other components cancel for the mythical spherical asteroid), the total impulse can be integrated, and the velocity perturbation obtained by dividing by the total mass

$$
\delta V=\frac{2 \pi R^{2} N \lambda \rho \nu \delta T_{\max }^{1 / 2}}{M_{\text {asteroid }}} \int_{1 / 1+x}^{1} \frac{(1+x) \alpha^{2}-\alpha}{1+(1+x)^{2}-2(1+x) \alpha} d \alpha
$$

where

$$
C_{s}(\theta)=v(\delta T(\theta))^{1 / 2}
$$


This impulse is based only on the momentum in the material that is heated to a gas and ejected from the asteroid. Momentum conservation requires that the asteroid have at least this momentum. Processes like compaction do not reduce this momentum, but will reduce velocity gradients near the expansion region assuring that the body shares the impulse. The integral limit is set by the horizon. For low altitudes, (small $x$ ) the heating is more intense, but the ejecta mass is small. At larger altitudes the reduced solid angle of the asteroid also leads to a drop-off. As a result, there is an optimum height of burst. Fortunately it is rather broad, eliminating any difficulties with triggering the burst at the proper time.

Fig 21 the impulse function versus altitude

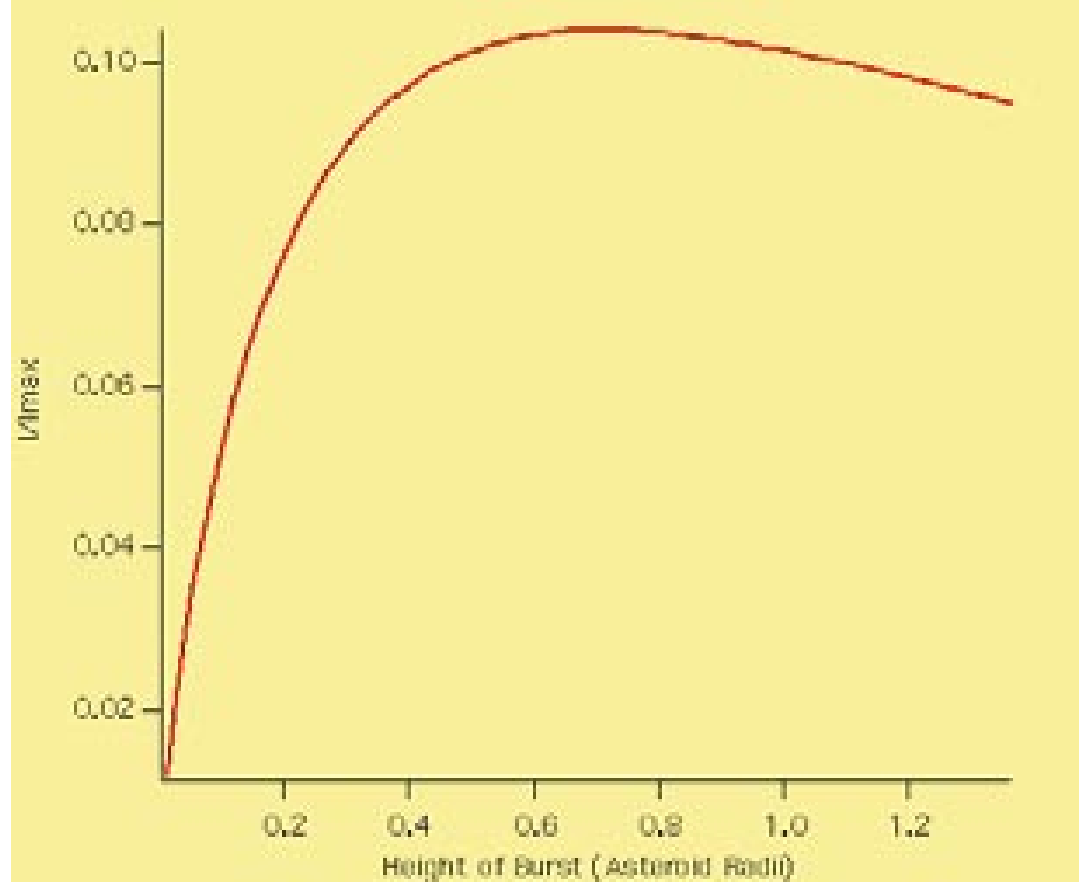

As a result, a kilometer diameter asteroid with density 2 will receive a velocity impulse of about $6.5 \mathrm{~cm} / \mathrm{s}$ from a device with a total yield near 1.5 megatons detonated about $300 \mathrm{~m}$ above the surface. Nuclear explosions of this magnitude are a well tested technology, including plowshare designs that emphasized fusion, and had a large neutron output. The Department of Energy office of science and technical information has photos and information on the Starfish Prime event of 9 July 1962 in which a 1.4 megaton device was detonated 400-kilometers above Johnson Island (http://www.osti.gov/historicalfilms/opentext/data/0800062.html).

The above discussion, has emphasized neutrons as the best war to produce a single impulse capable of changing the speed of a kilometer object by centimeters per second. A series of smaller impulses will be necessary to maintain the integrity of bodies that are only a couple of hundred meters diameter. There, where penetration depths of millimeters are sufficient, X-ray sources from lower yield nuclear explosives might be used, and MIRV technology used to produce a series of small taps to achieve the 
cumulative impulse required. While this is less efficient for weight, it should be considered as an even more conservative approach.

\section{Conclusions}

For the orbits typical of NEO's, speed changes of 1 to $10 \mathrm{~cm} / \mathrm{s}$ are sufficient to divert a body from impacting, with a warning time of years to decades. At present, nuclear devices offer a known technology for producing such velocity perturbations, with the demonstrated ability to be carried on missiles. When an object with an impacting orbit is found, nuclear devices offer an opportunity to divert disaster that requires only a few years to decades of reaction time.

In the probable circumstance that there is century of warning time, where the collision is known to be probable, the nuclear option allows time for reasoned action. A first obvious step is to learn about the structure of the threatening object. Again, technology for obtaining such information, like that proposed for Clementine II, has already been developed. Once the threat has been well defined, society can decide, based on the technology of the day, how to respond. As shown in the introductory quote, steam was the technology of the $19^{\text {th }}$ century, and nuclear energy is that of the $20^{\text {th }}$ and first part of the $21^{\text {st }}$.

As a final note, even the nuclear option will likely require some years of preparation. The optimal type of device may not be in any nations stockpile, and in the decades to come, there may not be stockpiles. Remanufacture could involve developing new processes for materials and parts that are not available. However the nuclear explosives are obtained, more than one must be available to limit the risk a failure. Some time may also be required to develop suitable boosters capability to deliver these devices to the optimal perturbation point. The most important current action is to vigilance so that we have a long-term prediction capability

\section{Acknowledgement:}

I wish to thank David Lynch, Don Yeomans, and Glenn Peterson for helpful discussions, as well as Peter Bedrossian for his neutronics calculation.

This work was performed under the auspices of the U.S. Department of Energy by University of California, Lawrence Livermore National Laboratory under Contract W7405-Eng-48.

\section{Cited references:}

J. D. Giorgini, S. J. Ostro, L. A. M. Benner, P. W. Chodas, S. R. Chesley, R. S. Hudson, M. C. Nolan, A. R. Klemola, E. M. Standish, R. F. Jurgens, R. Rose, A. B. Chamberlin, D. K. Yeomans, and J.-L. Margot Science Apr 5 2002: 132-136.

Carusi et. al. Icarus 2002 V159, p417-422

Glasstone and Dolan (1977)

Solem, JBIS, V53, p180,2000 